\title{
Women with a low-satiety phenotype show impaired appetite control and greater resistance to weight loss
}

\author{
Nicola J. Buckland ${ }^{1 *}$, Diana Camidge ${ }^{2}$, Fiona Croden ${ }^{2}$, Anna Myers ${ }^{3}$, Jacquelynne H. Lavin ${ }^{4}$, \\ R. James Stubbs ${ }^{2}$, John E. Blundell ${ }^{2}$ and Graham Finlayson ${ }^{2}$ \\ ${ }^{1}$ Department of Psychology, University of Sheffield, Cathedral Court, 1 Vicar Lane, Sheffield S1 2LT, UK \\ ${ }^{2}$ Human Appetite Research Unit, Appetite Control and Energy Balance Group, School of Psychology, University of Leeds, Leeds \\ LS2 9 TJT, UK \\ ${ }^{3}$ Sheffield Hallam University, Centre for Sport and Exercise Science, Collegiate Hall, Sheffield S1O 2BP, UK \\ ${ }^{4}$ Nutrition and Research Department, Slimming World, Alfreton, Derbyshire DE55 4RF, UK
}

(Submitted 8 February 2019 - Final revision received 5 July 2019 - Accepted 17 July 2019)

\section{Abstract}

This trial compared weight loss outcomes over 14 weeks in women showing low- or high-satiety responsiveness (low- or high-satiety phenotype (LSP, HSP)) measured by a standardised protocol. Food preferences and energy intake (EI) after low and high energy-density (LED, HED) meals were also assessed. Ninety-six women ( $n 52$ analysed; 41.24 (sD 12.54 ) years; 34.02 (sD 3.58 ) kg/m²) engaged in one of two weight loss programmes underwent LED and HED laboratory test days during weeks 3 and 12. Preferences for LED and HED food (Leeds Food Preference Questionnaire) and ad libitum evening meal and snack EI were assessed in response to equienergetic LED and HED breakfasts and lunches. Weekly questionnaires assessed control over eating and ease of adherence to the programme. Satiety quotients based on subjective fullness ratings post LED and HED breakfasts determined LSP ( $n$ 26) and HSP ( $n$ 26) by tertile splits. Results showed that the LSP lost less weight and had smaller reductions in waist circumference compared with HSP. The LSP showed greater preferences for HED foods, and under HED conditions, consumed more snacks (kJ) compared with HSP. Snack EI did not differ under LED conditions. LSP reported less control over eating and reported more difficulty with programme adherence. In conclusion, low-satiety responsiveness is detrimental for weight loss. LED meals can improve self-regulation of EI in the LSP, which may be beneficial for longer-term weight control.

Key words: Satiety phenotypes: Weight loss: Energy density: Food intake: Food preferences: Appetite control

In $2015,63 \%$ of UK adults were overweight or obese ${ }^{(1)}$. In efforts to control body weight, two thirds of women have reported a recent weight loss attempt ${ }^{(2)}$. Weight loss in response to such attempts varies ${ }^{(3)}$, and few individuals achieve long-term weight loss ${ }^{(4-6)}$. Individuals who have attempted weight loss report that hunger is one of the main challenges to losing weight ${ }^{(7,8)}$. As such the ability to detect appetite sensations may impact the success of a weight loss attempt.

There is variability in the extent to which individuals are able to detect changes in appetite sensations after eating ${ }^{(9,10)}$. The satiety quotient (SQ) has been used to measure the degree to which individuals feel sated in response to a meal (satiating efficiency) (meals are often calibrated to the estimated individual daily energy needs $\left.{ }^{(11)}\right)$. The SQ measures changes in subjective appetite sensations following a fixed-energy meal. Higher SQ scores (greater satiating efficiency) have been found to correspond with lower energy intake (EI) in laboratory and free-living settings ${ }^{(12,13)}$. Based on SQ scores, individuals can be categorised as either low- or high-satiety behavioural phenotypes (LSP, HSP) ${ }^{(11,14,15)}$. These satiety phenotypes have been shown to differ on psychological ${ }^{(11,14)}$, metabolic ${ }^{(14)}$ and behavioural outcomes ${ }^{(11)}$. For instance, compared with the HSP, the LSP is associated with greater trait disinhibition (tendency to eat opportunistically $)^{(10,11)}$, lower craving control, greater preferences to eat high-fat foods (as indicated in the Leeds Food Preference Questionnaire (LFPQ) ${ }^{(16)}$ ) and greater meal EI ${ }^{(11)}$. As such, the evidence suggests that the LSP are less able to control their appetite and are susceptible to overconsumption compared with HSP.

Therefore, it is important to identify strategies that promote satiety in the LSP and prevent overconsumption. Low energydensity (LED) foods have been identified as a food associated

Abbreviations: EI, energy intake; HED, high energy density; HSP, high-satiety phenotype; ITT, intention to treat; LED, low energy density; LFPQ, Leeds Food Preference Questionnaire; LSP, low-satiety phenotype; SQ, satiety quotient; VAS, visual analogue scale.

* Corresponding author: Dr N. J. Buckland, email n.buckland@sheffield.ac.uk 
with increased satiation and satiety ${ }^{(17-19)}$. It is unknown whether LED meals improve LSP's acute appetite control; to date, studies have only compared LSP's and HSP's appetite responses to one meal ${ }^{(11,14)}$. To our knowledge, no studies have compared appetite responses to LED and high energy-dense (HED) meals in the satiety phenotypes. In terms of appetite responses in women engaged in weight loss, it is important to assess not only subjective appetite and intake but also implicit preferences for high-fat food. Dietary energy reductions have been shown to increase the rewarding value of and appeal for food ${ }^{(20,21)}$, which may impair dietary control. It is currently unknown whether LED foods can prevent such hedonic motivations previously found in the $\operatorname{LSP}^{(11)}$.

Moreover, the impact of the LSP on weight loss is unclear. One study in men reported that the LSP lost less body weight after a 16-week diet compared with the $\mathrm{HSP}^{(15)}$. Whereas another study using male and female participants reported no effects of the LSP on weight change ${ }^{(22)}$. As such, further studies, which investigate specific samples (e.g. women only) and types of weight loss programmes followed, are needed to confirm the role that LSP has on weight loss

The present study characterised women as LSP or HSP and compared weight loss and changes in body composition after a 14-week weight loss programme (Slimming World, UK or NHS Live Well programme). Food intake and food preferences (liking and wanting) in response to LED and HED meals in LSP and HSP were also assessed in the laboratory. Additionally, the study compared LSP's and HSP's self-reported appetite control during the programme. It was hypothesised that compared with the HSP, the LSP would lose less body weight and body fat, have smaller reductions in waist and hip circumference, exhibit weaker appetite control under HED test conditions compared with LED test conditions and report weaker appetite control during the programme.

\section{Methods}

\section{Participants}

The study was conducted as a secondary analysis from data collected for a trial that is reported in more detail elsewhere ${ }^{(19)}$ (ClinicalTrials.gov no. NCT02012426). The present analysis differs from the previous analyses (which reported effects for the overall sample), by focusing specifically on satiety phenotypes. Based on previous research ${ }^{(15)}$, power calculations in $G^{*}$ Power with an $\alpha$ of 0.05 and power of 0.80 showed that a sample size of fifty-four participants would be sufficient to detect significant differences in weight change between satiety phenotypes ${ }^{(23)}$. Ninety-six women who were overweight or obese and had recently enrolled in a weight loss programme were recruited. Participants were recruited from Slimming World, UK groups ${ }^{(24)}$ ( $n$ 49) and the University of Leeds population and local area ( $n$ 47). Only volunteers who had recently enrolled in the Slimming World, UK programme were recruited to the Slimming World arm of the trial. Following recruitment, this group continued with the Slimming World, UK programme. Participants recruited from the University of Leeds and local area followed the NHS Live Well programme ${ }^{(25)}$. Further details about each programme have been previously reported ${ }^{(19)}$. In brief, Slimming World, UK, is a group-based commercial weight management programme. The programme advocates ad libitum intake of LED foods and controlled amounts of higher energydense foods. The NHS Live Well programme is an online programme that recommends a daily $600 \mathrm{kcal}(2510 \mathrm{~kJ})$ deficit and provides dietary and physical activity advice.

Volunteers who indicated confounding health issues, were taking medications that affect appetite or weight, had received bariatric surgery and indicated an inability to eat the study foods or follow study procedures were excluded (for full exclusion criteria see Buckland et al. $\left.{ }^{(19)}\right)$. The study was approved by the University of Leeds, School of Psychology ethics committee. Participants provided written informed consent and received $£ 250$ upon study completion.

\section{Design, measures and procedure}

At week 1, body weight and height were measured (by a Slimming World, UK group leader or University researcher using a stadiometer and electronic scales) and participants started their weight loss programme. During weeks 2 and 14, participants attended a morning session at the University of Leeds, Human Appetite Research Unit, and under standardised controlled procedures (overnight fast, 24-h alcohol abstinence and no physical activity on the morning of the session; compliance was checked upon arrival) the following measures were assessed: body weight and body composition (body fat, percentage body fat and fat-free mass assessed using air plethysmography (Bodpod, Concord) in minimal clothing), waist and hip circumference (measured by researcher, average of two measures), RMR (indirect calorimeter, GEM; Nutren Technology Ltd), resting blood pressure and heart rate (Omron M10-IT digital blood pressure cuff) and psychometric traits (cognitive restraint, trait disinhibition and trait hunger using the Three-Factor Eating Questionnaire $\left.{ }^{(26)}\right)$. Other measures, specifically relevant to the larger study, were also recorded but not reported here ${ }^{(19)}$.

To assess appetite control in response to energy-density manipulations, early on in the programme (week 3), participants attended the unit under standardised controlled procedures mentioned above (but with instructions to maintain similar levels of physical activity across days), and in a repeated-measure design was provided with LED or HED meals. Condition order was counterbalanced across participants, and each condition was separated by a minimum of $7 \mathrm{~d}$ in both weeks 3 and $12^{(27)}$. The energy-density manipulations were repeated later on in the programme (week 12). During the interval between conditions (both at the early late phase of the programme), participants completed weighed food diaries and wore a physical activity monitor (SenseWear Armband; BodyMedia, Inc.) which assessed total physical activity and sleep duration, as has previously been described ${ }^{(28)}$. The number of days between participants starting the weight loss programme and completing the measures session and test meal probe days were matched across programme type. Thus, participants from the Slimming World, UK and NHS Live Well programme had been engaged in a weight loss programme for the same duration when body weight and body composition (mean $21 \pm 6 \mathrm{~d}$ ) and appetite 
control (mean $27 \pm 7$ d) were assessed. A diagram of the overall study timeline has been reported here ${ }^{(19)}$.

\section{Energy density}

On test meal days, participants were provided with either a day of LED $(\leq 0.8 \mathrm{kcal} / \mathrm{g} ; \leq 3.3 \mathrm{~kJ})$ or HED foods $(\geq 2.5 \mathrm{kcal} / \mathrm{g}$; $\geq 10 \cdot 5 \mathrm{~kJ}$ ) across breakfast, lunch, an evening meal and evening snacks. Across both LED and HED conditions, the breakfast and lunch provided $50 \%$ of total daily energy needs (based on RMR $\times$ 1.4 sedentary physical activity levels). The evening meal and evening snacks were served ad libitum (for more details see Buckland et $\left.a l .{ }^{(19)}\right)$. Food were sourced from a UK supermarket except for the LED evening meal (beef chilli con carne) which was provided by Slimming World, UK and used in all LED test sessions (regardless of the weight loss programme being followed). Energy density was manipulated using LED and HED versions of products. For fixed meals, participants were required to eat the entire portion. For the evening meal, participants were instructed to help themselves with as much or as little of the food as they liked and to eat until they felt they had eaten enough. For snacks, participants were instructed to help themselves to as much or as little of the foods as they liked, to avoid eating other foods and to avoid sharing the snacks. Meals were served $4 \mathrm{~h}$ apart and took place in the research unit. Participants could leave the research unit between meals but were instructed to fast and consume water only during this period. Bottled water was provided to improve compliance. After each meal, participants rated meal palatability (appeal, pleasantness and satisfaction) on 100-mm visual analogue scale (VAS). Participants took snacks home and returned left over packaging the next day, so that intake could be assessed.

\section{Food intake and food preferences}

To determine food intake, meals were covertly weighed pre- and post-consumption. Weight intake was converted to EI using food composition tables ${ }^{(29)}$ and manufacturers' nutritional information. Meal and snack intake were summed to provide the total day intake.

Implicit and explicit food preferences to LED and HED food were assessed pre- and post-lunch using the validated LFPQ (for details see Finlayson et al. $\left.{ }^{(16)}\right)$. Participants were presented with sweet and savoury, LED and HED foods on screen, and to assess explicit liking, participants rated the pleasantness of each food. To assess implicit wanting, participants completed a forcedchoice task whereby the food images were paired, so that every image from each of the four food types (LED/HED and sweet/ savoury) were compared with every other type over repeated trials (food pairs). Participants were instructed to respond as quickly and accurately as possible to indicate the food they most wanted to eat at that time. Reaction times were recorded and used to compute mean response times for each food type after adjusting for frequency of selection. The mean LED food scores were subtracted from the mean HED food scores to provide a bias score for HED $v$. LED foods. Higher scores indicate greater preference for HED relative to LED foods.

\section{Satiety quotient}

During the LED and HED test meal days, participants rated subjective fullness sensations on 100-mm VAS immediately pre and post each meal and at hourly intervals ('How hungry do you feel right now', ' $0=$ not at all'; ' $100=$ extremely' $^{(27)}$. The SQ was calculated using the average fullness scores collected at prebreakfast and 180-min post-breakfast on the LED and HED probe days administered in the early phase of the programme. Fullness ratings were used because of the appetite sensations (e.g. hunger, desire to eat), fullness is the strongest predictor of EI, and it has been argued that fullness is the easiest sensation to detect due to its links with physical gastric distension ${ }^{(12)}$. Tertile splits were conducted on appetite ratings recorded on the early probe days only to prevent weight loss over the programme confounding the satiety phenotype categorisation. (SQ scores obtained at the early (HSP: 12.64 (sD 3.40); LSP: 1.05 (SD 2.76)) and late phases of the programme (HSP: 9.59 (sD 6.16); LSP: 4.61 (sD 5.79)) were significantly correlated, $r 0.44, P=0.001$.) There was good internal reliability between scores (Cronbach's $\alpha=0 \cdot 65$ ). The SQ was calculated using the following formula:

$\mathrm{SQ}(\mathrm{mm} / \mathrm{kcal})=\left(\frac{180-\text { min post }- \text { breakfast fullness }(\mathrm{mm})-\text { fasting fullness }(\mathrm{mm})}{\text { Breakfast EI }(\mathrm{kcal})}\right) \times 100$

\section{Appetite control during the programme}

Self-reported appetite control was assessed outside the unit, with questionnaires each week. Participants were instructed to complete questionnaires on the same day and time each week. Participants rated control over eating, ability to adhere to the programme's food choices, adherence to the overall programme and the ease of adhering to the programme on 100-mm VAS ('How much do you feel IN CONTROL of what you're eating?' 'Have you felt able to stick to your plan regarding your food choices?' 'How WELL have you managed to stick with the weight control programme?' 'How EASY do you find it to stick to your weight control programme?').

\section{Statistical analyses}

Raw SQ scores from the early probe days in the full sample were initially included as a covariate in an ANCOVA, examining changes in body weight between weeks 1 and 14 controlling for programme type. The week $\times S Q$ interaction was significant, $P=0 \cdot 003, \eta p^{2}=0 \cdot 11$, and as such further analyses of SQ (comparisons of LSP and HSP) were conducted using point estimates of lower and upper tertile SQ scores. Scores $<4.5$ were classified as LSP; scores $>8.5$ were classified as HSP. These cut-off points are similar to those used in previous research ${ }^{(14)}$. Participants scoring 4.6-8.5 were unclassified and not included in further analyses or figures to facilitate interpretation and visualisation of findings.

Outcomes were assessed in participants who completed the study with eligible data (completers analysis). For body weight and body composition outcomes, separate intention-to-treat (ITT) analyses using the last observation carried forward were 
also conducted to account for participants who did not complete the study, provided that data were available (no data were available for participants who withdrew before completing the early test meal sessions) ${ }^{(30)}$. To assess data collected from the SenseWear armbands, proprietary algorithms available in the SenseWear software were used (SenseWear Professional software version 8.0, algorithm version 5.2). Total physical activity was calculated by summing the amount of time spent in activities $>1.5$ MET.

A $\chi^{2}$ test showed that participants from each weight loss programme were evenly distributed across the satiety phenotypes (LSP: Slimming World $n$ 12, NHS Live Well $n$ 14; HSP: Slimming World $n$ 13, NHS Live Well $n 13 ; \chi^{2}(1)=0.78$, $P=0 \cdot 78)$. Programme type and percentage weight change up to the week 2 measure session was included as a covariate in all analyses except for $t$ tests and unless specified. For concision, results are reported for covariates only when covariates were significant.

To compare the characteristics of the satiety phenotypes at week 1, ANCOVA were conducted. Mixed ANCOVA were used to compare changes in body weight and composition between satiety phenotypes. To control for starting body weight and composition, percentage change in body weight outcomes between satiety phenotypes was compared. Mixed ANCOVA were used to compare food intake and food preferences in the satiety phenotypes under LED and HED conditions. To assess appetite control during the programme, mixed ANOVA were used to compare ratings between satiety phenotypes across weeks. Significant interactions were explored with $t$ tests unless specified. Averages from early and late probe days were computed where necessary. Results were considered significant if $P<0.05$ except for tests with multiple comparisons, whereby a more conservative $P$ value was used to account for multiple comparisons (0.05 divided by the number of comparisons). The analysis reports comparison results between LSP and HSP only. Overall changes over weeks for each outcome have previously been reported for the full sample ${ }^{(19)}$. Data are presented as means, standard deviations and $95 \%$ CI unless specified. For concision, when multiple results are reported, the most conservative $P$ value is provided. Partial eta square $\left(\eta p^{2}\right)$ is reported for effect sizes and interpreted as 0.01 small, 0.06 moderate and $0 \cdot 14$ large $\mathrm{e}^{(31)}$. All statistical analyses were based on kcal and the values reported are in $\mathrm{kJ}$ using the conversion $1 \mathrm{kcal}$ $=4 \cdot 184$. Analyses were conducted in Statistical Package for Social Science (IBM SPSS, version 24).

\section{Results \\ Sample characteristics}

Of the ninety-six participants (age 41.24 (SD 12.54) years; BMI 34.02 (sD 3.58$) \mathrm{kg} / \mathrm{m}^{2}$ ), ten withdrew and six were excluded (ineligible $n 3$, extreme weight gain $n 1$, broken leg $n 1$; medical condition $n 1$ ). Of the three ineligible, two were long-term members of Slimming World, UK and led group sessions, and one had a confounding health issue identified after study enrolment. One participant could not be classified to a satiety phenotype due to missing appetite ratings. The remaining seventy-nine participants were classified as LSP ( $n$ 26), HSP ( $n$ 26) or unclassified ( $n$ 27). Data from four other participants were available for ITT analyses (LSP $n 2$, HSP $n$, unclassified $n$ 1).

Baseline characteristics for the LSP and HSP that completed the trial are shown in Table 1. By definition, the LSP's SQ was significantly lower compared with the HSP. With the exception of blood pressure, no baseline outcomes significantly differed between satiety phenotypes. The LSP had significantly greater resting systolic and diastolic blood pressure that remained significant when controlling for body weight and BMI.

\section{Changes in body weight and body composition}

Results for changes in body weight and body composition did not differ between completers and ITT analyses unless stated (see Table 2). The HSP lost significantly more weight compared with the LSP as qualified by a significant week $\times$ phenotype interaction on body weight $\left(P=0.02, \eta p^{2}=0 \cdot 10\right)$ (approached significance in the ITT model, $P=0.09$, $\left.\eta p^{2}=0.05\right)$. (Percentage weight change at week 2 was a significant predictor of weight change at week $14(\%)(P<0 \cdot 001$, $\left.\eta p^{2}=0 \cdot 40\right)$. Greater weight loss at week 2 was associated with significantly greater weight loss at week $14(r$ 0.71, $P<0 \cdot 001)$.)

For body composition outcomes, data were missing for eight participants due to a technical fault (LSP $n$ 7). In response to the technical fault, four participants' (LSP $n 1$ ) data were collected in weeks 1 and 14 with bioelectrical impedance (model BC418MA, Tanita) and due to the consistent method of assessment in both weeks the data were retained in the analysis. Changes in fat mass and \%fat did not significantly differ between satiety phenotypes $\left(P=0 \cdot 16, \eta p^{2}=0 \cdot 05\right)$. (Percentage weight change at week 2 was a significant covariate of changes in percentage body fat (completers and ITT) and body fat mass at week 14 (ITT only). Greater weight loss at week 2 was associated with greater reductions in body fat mass and percentage body fat at week 14 ( $r$ 42, $P=0.004)$.) In completers, there was a significant week $\times$ satiety phenotype interaction on fat-free mass $\left(P=0 \cdot 04, \eta p^{2}=0 \cdot 10\right)$ (non-significant for ITT, $P=0.09, \eta p^{2}=0.06$ ), but post boc comparisons did not reveal any significant differences between phenotypes $(P=0 \cdot 06)$. Waist reductions were significantly greater for the HSP compared with the LSP (week $\times$ satiety phenotype interaction on waist circumference, $P=0.02$, $\left.\eta p^{2}=0 \cdot 12\right)$ and remained significant when controlling for starting waist circumference $\left(P=0 \cdot 02, \eta p^{2}=0 \cdot 13\right)$. Changes in hip circumference did not significantly differ between satiety phenotypes $\left(P=0 \cdot 10, \eta p^{2}=0 \cdot 06\right)$.

\section{Food intake and food preferences}

Snack and total day intake data were missing for two participants due to non-returned snacks (LSP $n$ 1). The LSP's and HSP's mean EI for fixed meals, evening meals and evening snack are shown in Fig. 1. Evening meal and total day EI did not significantly differ between satiety phenotypes $\left(P=0.07, \eta p^{2}=0 \cdot 07\right)$, but LSP's snack EI was significantly greater compared with the HSP $\left(P=0.02, \eta p^{2}=0 \cdot 11\right)$. There was a significant condition $\times$ satiety phenotype interaction on snack intake $\left(P=0 \cdot 04, \eta p^{2}=0 \cdot 09\right)$, 
Table 1. Baseline characteristics for the low-satiety phenotype (LSP) and high-satiety phenotype (HSP)

(Mean values, standard deviations and $95 \%$ confidence intervals)

\begin{tabular}{|c|c|c|c|c|c|c|c|c|}
\hline & \multicolumn{3}{|c|}{ LSP $(n 26)$} & \multicolumn{3}{|c|}{$\operatorname{HSP}(n 26)$} & \multirow[b]{2}{*}{ LSP $v$. HSP: $P$} & \multirow[b]{2}{*}{$\eta p^{2}$} \\
\hline & Mean & SD & $95 \% \mathrm{Cl}$ & Mean & SD & $95 \% \mathrm{Cl}$ & & \\
\hline $\mathrm{SQ}(\mathrm{mm} / \mathrm{kcal})$ & 1.05 & $2 \cdot 76$ & $-0 \cdot 06,2 \cdot 16$ & $12 \cdot 64$ & 3.40 & $11.27,14.02$ & $<0.001$ & 0.77 \\
\hline Week 2 weight change $(\%) \dagger$ & $-2 \cdot 12$ & 1.64 & $-2 \cdot 79,-1 \cdot 46$ & -2.97 & 1.60 & $-3 \cdot 61,-2 \cdot 32$ & 0.06 & 0.07 \\
\hline Age (years) & 39.31 & 11.33 & $34.73,43.88$ & 44.54 & $12 \cdot 06$ & $39.67,49.41$ & 0.14 & 0.05 \\
\hline Height $(m)$ & 1.65 & 0.06 & $1 \cdot 63,1.68$ & 1.63 & 0.08 & $1 \cdot 60,1.66$ & 0.43 & 0.01 \\
\hline Weight (kg) & 94.42 & $13 \cdot 39$ & $89.02,99.83$ & 90.99 & $13 \cdot 72$ & $85 \cdot 36,96 \cdot 44$ & 0.56 & 0.01 \\
\hline BMI $\left(\mathrm{kg} / \mathrm{m}^{2}\right)$ & 34.41 & 3.61 & $32 \cdot 95,35 \cdot 86$ & 33.99 & 3.40 & $32 \cdot 61,35.36$ & 0.84 & 0.01 \\
\hline Fat mass $(\mathrm{kg}) \ddagger$ & 43.52 & 11.50 & $37.98,49 \cdot 07$ & $40 \cdot 92$ & $9 \cdot 60$ & $36 \cdot 96,44 \cdot 88$ & 0.35 & 0.02 \\
\hline$\%$ Fatł & 45.89 & 6.97 & $42 \cdot 54,49 \cdot 25$ & $45 \cdot 92$ & 4.59 & $44.02,47.81$ & 0.81 & 0.01 \\
\hline FFM (kg)‡ & $50 \cdot 25$ & 6.58 & $47 \cdot 08,53.42$ & $47 \cdot 34$ & 5.69 & $45.00,49.69$ & 0.35 & 0.02 \\
\hline RMR (kcal/d) & 1750 & 280 & 1637,1863 & 1628 & 243 & 1533,1722 & 0.23 & 0.03 \\
\hline Waist $(\mathrm{cm}) \S$ & $109 \cdot 64$ & $13 \cdot 36$ & $104 \cdot 12,115 \cdot 15$ & $108 \cdot 21$ & $11 \cdot 26$ & $103 \cdot 46,112 \cdot 97$ & 0.73 & 0.01 \\
\hline Hip (cm) & $118 \cdot 12$ & $11 \cdot 15$ & $113 \cdot 61,122 \cdot 62$ & $116 \cdot 75$ & $10 \cdot 23$ & $112 \cdot 61,120 \cdot 80$ & 0.99 & 0.00 \\
\hline Systolic BP $(\mathrm{mmHg}) \|$ & 122.44 & $13 \cdot 71$ & $116.91,127.98$ & 111.76 & $12 \cdot 15$ & $106 \cdot 74,116 \cdot 78$ & $0.01^{*}$ & $0 \cdot 13$ \\
\hline Diastolic BP $(\mathrm{mmHg}) \|$ & 84.29 & $11 \cdot 16$ & $79 \cdot 78,88 \cdot 80$ & $75 \cdot 58$ & 8.63 & $72 \cdot 02,79 \cdot 14$ & $0.01^{*}$ & 0.14 \\
\hline Heart rate $(\mathrm{bpm})$ ๆ & 63.96 & 8.35 & $60 \cdot 52,67 \cdot 40$ & $61 \cdot 82$ & $9 \cdot 12$ & $58.05,65.59$ & 0.69 & 0.01 \\
\hline Fasting glucose & 4.84 & 0.78 & $4.51,5 \cdot 16$ & 4.90 & 0.64 & $4 \cdot 64,5 \cdot 17$ & 0.81 & 0.01 \\
\hline TFEQ restraint & 9.50 & $3 \cdot 17$ & $8 \cdot 22,10 \cdot 78$ & 8.69 & 3.33 & $7.35,10.04$ & 0.15 & 0.04 \\
\hline TFEQ disinhibition & 10.54 & 3.18 & $9.25,11.82$ & 9.92 & 2.92 & $8 \cdot 74,11 \cdot 10$ & 0.99 & 0.00 \\
\hline TFEQ hunger & $7 \cdot 23$ & 3.54 & $5.80,8.66$ & 5.96 & $3 \cdot 14$ & $4.69,7 \cdot 23$ & 0.50 & 0.01 \\
\hline
\end{tabular}

$\mathrm{SQ}$, satiety quotient; FFM, fat-free mass; BP, blood pressure; TFEQ, Three-Factor Eating Questionnaire.

* Significantly different from LSP, controlling for week 1 body weight and BMI $(P<0.05)$.

† Week 2 weight change refers to percentage weight change since starting the weight loss programme and the measures session completed in week 2.

† LSP $n$ 19; HSP $n 25$.

§ LSP $n$ 25; HSP $n 24$.

\| HSP $n$ 25; comparisons controlled for weight loss programme and percentage weight change at week 2.

I LSP $n$ 25; HSP $n 25$.

Table 2. Changes in study outcomes for the low- and high-satiety phenotypes in completers and last observation carried forward (LOCF) analyses* (Mean values, standard deviations and $95 \%$ confidence intervals)

\begin{tabular}{|c|c|c|c|c|c|c|c|c|c|}
\hline & \multirow[b]{2}{*}{$n$} & \multicolumn{3}{|c|}{ Low-satiety phenotype } & \multicolumn{3}{|c|}{ High-satiety phenotype } & \multirow[b]{2}{*}{$P$} & \multirow[b]{2}{*}{$\eta p^{2}$} \\
\hline & & Mean & SD & $95 \% \mathrm{Cl}$ & Mean & SD & $95 \% \mathrm{Cl}$ & & \\
\hline \multicolumn{10}{|c|}{$\%$ Weight change } \\
\hline Completers & 52 & $-3 \cdot 11$ & 3.43 & $-4 \cdot 49,-1 \cdot 72$ & $-6 \cdot 35$ & $4 \cdot 23$ & $-8.05,-4.64$ & 0.02 & 0.10 \\
\hline LOCF & 55 & $-3 \cdot 19$ & 3.39 & $-4.53,-1.85$ & -5.88 & 4.50 & $-7 \cdot 63,-4 \cdot 14$ & 0.08 & 0.06 \\
\hline \multicolumn{10}{|l|}{ Weight (kg) } \\
\hline Completers & 52 & $-2 \cdot 89$ & 3.08 & $-4 \cdot 13,-1 \cdot 64$ & $-5 \cdot 71$ & $3 \cdot 65$ & $-7 \cdot 19,-4 \cdot 23$ & 0.02 & 0.10 \\
\hline LOCF & 55 & $-2 \cdot 97$ & 3.04 & $-4 \cdot 17,-1 \cdot 76$ & $-5 \cdot 28$ & 3.93 & $-6 \cdot 80,-3 \cdot 76$ & 0.08 & 0.06 \\
\hline \multicolumn{10}{|l|}{ Fat mass $(\mathrm{kg})$} \\
\hline Completers & $44 \dagger$ & -0.91 & $2 \cdot 02$ & $-1.88,0.07$ & -2.69 & $3 \cdot 19$ & $-4.01,-1.37$ & NS & 0.01 \\
\hline LOCF & $47 \dagger$ & -0.93 & 1.97 & $-1.85,-0.01$ & $-2 \cdot 28$ & 3.42 & $-3.63,-0.93$ & NS & 0.01 \\
\hline \multicolumn{10}{|l|}{ Percentage fat } \\
\hline Completers & $44 \dagger$ & -0.64 & 1.41 & $-1.32,0.04$ & $-1 \cdot 60$ & 2.68 & $-2.71,-0.49$ & NS & 0.01 \\
\hline LOCF & $47 \dagger$ & -0.60 & 1.38 & $-1.25,0.04$ & -1.35 & 2.75 & $-2.44,-0.26$ & NS & 0.01 \\
\hline \multicolumn{10}{|c|}{ Fat-free mass (kg) } \\
\hline Completers & $44 \dagger$ & 0.22 & $1 \cdot 20$ & $-0.36,0.79$ & -0.42 & 1.09 & $-0.88,0.03$ & 0.04 & 0.10 \\
\hline LOCF & $47 \dagger$ & $0 \cdot 13$ & $1 \cdot 23$ & $-0.45,0.70$ & -0.39 & 1.08 & $-0.82,0.04$ & NS & 0.06 \\
\hline \multicolumn{10}{|c|}{$\begin{array}{l}\text { Waist circumference } \\
(\mathrm{cm})\end{array}$} \\
\hline Completers & $49 \ddagger$ & -0.66 & 3.97 & $-2.30,0.98$ & -3.30 & $2 \cdot 84$ & $-4 \cdot 50,-2 \cdot 10$ & $0.01 \S$ & 0.13 \\
\hline LOCF & 49 & -0.66 & 3.97 & $-2 \cdot 30,0.98$ & $-3 \cdot 30$ & $2 \cdot 84$ & $-4 \cdot 51,-2 \cdot 10$ & $0.01 \S$ & 0.13 \\
\hline \multicolumn{10}{|c|}{$\begin{array}{l}\text { Hip circumference } \\
(\mathrm{cm})\end{array}$} \\
\hline Completers & 52 & -0.21 & 4.86 & $-2 \cdot 18,1 \cdot 75$ & $-2 \cdot 54$ & 4.28 & $-4 \cdot 27,-0 \cdot 81$ & NS & 0.06 \\
\hline LOCF & 55 & 0.28 & 4.78 & $-1 \cdot 61,2 \cdot 17$ & $2 \cdot 19$ & 4.33 & $0.51,3.87$ & NS & 0.04 \\
\hline
\end{tabular}

* Negative values indicate decreases between weeks. All comparisons controlled for weight loss programme (Slimming World, UK or NHS Live Well programme) and weight change at week $2(\%)$.

† For fat mass, percentage fat mass and fat-free mass, data were missing from eight participants due to a fault in the BodPod.

$\ddagger$ Missing data from three participants due to measurement issues (low satiety phenotype $n 1$ ).

$\S$ Remained significant when controlling for starting waist circumference $(P<0.05)$. 
Table 3. Self-reported appetite control during the programme for the low- and high-satiety phenotypes* (Mean values with their standard errors, and $95 \%$ confidence intervals)

\begin{tabular}{|c|c|c|c|c|c|c|c|c|}
\hline & \multicolumn{3}{|c|}{ Low-satiety phenotype } & \multicolumn{3}{|c|}{ High-satiety phenotype } & \multirow[b]{2}{*}{$P$} & \multirow[b]{2}{*}{$\eta p^{2}$} \\
\hline & Mean & SEM & $95 \% \mathrm{Cl}$ & Mean & SEM & $95 \% \mathrm{Cl}$ & & \\
\hline on & $50 \cdot 3$ & 4.6 & $40 \cdot 9,59 \cdot 7$ & $73 \cdot 0$ & $4 \cdot 7$ & $63 \cdot 4,82 \cdot 7$ & 0.01 & 0.19 \\
\hline Have you felt able to stick to your plan regarding & $43 \cdot 6$ & $4 \cdot 1$ & $35 \cdot 3,51 \cdot 9$ & 61.9 & $4 \cdot 2$ & $53.4,70.5$ & 0.01 & 0.18 \\
\hline How WELL have you managed to stick to the programme? & $39 \cdot 8$ & 4.3 & $31 \cdot 0,48 \cdot 6$ & $60 \cdot 1$ & 4.4 & $51 \cdot 0,69 \cdot 1$ & 0.01 & 0.18 \\
\hline How EASY do you find it to stick to your weight control programme? & $46 \cdot 6$ & $4 \cdot 8$ & $36 \cdot 8,56 \cdot 4$ & $66 \cdot 0$ & $5 \cdot 0$ & $55 \cdot 9,76 \cdot 1$ & 0.05 & 0.12 \\
\hline
\end{tabular}

* Data missing for seventeen participants due to non-returned questionnaires; total sample size $n 35$ (low-satiety phenotype, $n$ 18). Responses ranged from ' $0=$ not at all' to ' 100 = very'. All comparisons controlled for weight loss programme (Slimming World, UK or NHS Live Well programme) and weight change at week 2 (\%).

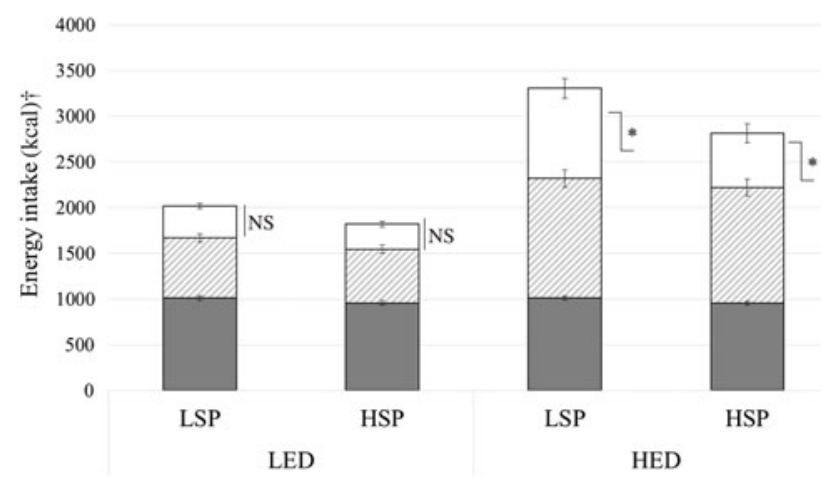

Fig. 1. Energy intake under low and high energy-density (LED, HED) conditions in the low- and high-satiety phenotypes (LSP, HSP). Values are means, with their standard errors represented by vertical bars. ${ }^{\star} P<0.05$ between LSP and HSP. $\square$, Fixed meals; $\square$, evening meals; $\square$, snacks. † To convert kcal to $\mathrm{kJ}$, multiply by $4 \cdot 184$.

which showed that under LED conditions, LSP's snack EI did not differ from HSP's snack EI (mean difference 63 (sEm 43; $95 \%$ CI $24,149) \mathrm{kcal}(264$ (SEM 180; $95 \%$ CI 100, 623) kJ); $P=0 \cdot 15$ ). However, under HED conditions, LSP's snack intake was 289 (SEM 133; $95 \%$ CI 22, 556) kcal (1209 (SEM 556; $95 \%$ CI 92, $2326) \mathrm{kJ}$ ) higher compared with HSP's snack intake $(P=0.03)$.

For gram intake, snack, evening meal and total day gram intake did not differ between satiety phenotypes $(P=0.05$, $\left.\eta p^{2}=0 \cdot 08\right)$. There was a significant condition $\times$ satiety phenotype interaction on evening meal gram intake $(P=0.003$, $\left.\eta p^{2}=0 \cdot 17\right)$ but post hoc comparisons failed to reach significance $(P=0 \cdot 16)$. No other condition $\times$ satiety phenotypes interactions on gram intake were significant and there were no significant covariates for gram intake.

For food preferences, independent of programme type, the LSP showed a greater fat bias for HED foods compared with the HSP who showed a greater bias for LED foods $(P=0.007$, $\eta p^{2}=0 \cdot 18$ (explicit liking: LSP, 9.01 (SEM 3.48; $95 \%$ CI 1.96, 16.06), HSP, -5.98 (sem 3.57; $95 \%$ CI -13.20, 1.25); implicit wanting: LSP, $17 \cdot 10$ (SEM 7.08; $95 \%$ CI $2 \cdot 76,31 \cdot 44$ ), HSP, -14.26 (SEM $7 \cdot 26 ; 95 \% \mathrm{CI}-28 \cdot 95,0 \cdot 44))$ ). (Programme type was a significant covariate for liking and wanting $\left(P=0 \cdot 03, \eta p^{2}=0 \cdot 12\right)$.)

Breakfast and lunch meal palatability ratings did not differ between the satiety phenotypes $\left(P=0 \cdot 23, \eta p^{2}=0 \cdot 03\right)$. Across conditions, the LSP rated the evening meals as less appealing, less pleasant and less filling compared with the HSP $(P=0.03$, $\eta p^{2}=0 \cdot 10$ ) (programme type was a significant covariate for appeal and pleasantness, $\left.P=0.03, \eta p^{2}=0.09\right)$. Satisfaction ratings for the ad libitum evening meal did not differ between phenotypes $\left(P=0.09, \eta p^{2}=0.06\right)$ (programme type was a significant covariate of evening meal satisfaction, $P=0 \cdot 04, \eta p^{2}=0 \cdot 09$ ) (see online Supplementary Table S1).

\section{Appetite control during the programme}

Compared with the HSP, the LSP felt significantly less in control over what they were eating, less able to adhere to the programme generally and to the food choices encouraged by the programme and found the programme more difficult to follow (see Table 3).

\section{Food diaries, sleep and physical activity}

Analysis of the food diaries completed at the start and end of the programme showed EI did not differ between satiety phenotypes (LSP, 6881 (SEM 322; $95 \%$ CI 6233, 7530) kJ/d; HSP, 6254 (sem 322; $95 \%$ CI 5606, 6902) kJ/d, $n$ 25, $P=0 \cdot 18$, $\eta p^{2}=0 \cdot 04$ ). (Food diary data $n 50$, missing data due to nonreturned diaries (LSP $n$; HSP $n$ 1).) Analysis of the physical activity monitors worn at the start and end of the programme also showed that sleep duration (LSP, 7.06 (SEM 0.19; $95 \%$ CI 6.67, 7.45) h/d; HSP, 6.97 (SEM 0.17; $95 \%$ CI 6.63, 7.32) h/d, $\left.P=0.73, \eta p^{2}=0.003\right)$ and total physical activity did not differ between phenotypes (LSP, 4.29 (SEm 0.45; $95 \%$ CI 3.38, 5.21) h/d; HSP, 4.65 (SEM 0.39; $95 \%$ CI 3.85, 5.45) h/d, $P=0.56$, $\eta p^{2}=0 \cdot 01$ ). (Physical activity and sleep total $n 39$ participants (LSP $n$ 17)). Missing physical activity and sleep data due to invalid data ( $<5 \mathrm{~d}$ (including $<1$ weekend day) ( $n 11)$ or technical issues $(n$ 2).)

\section{Discussion}

In the present study of a 14-week weight management programme, the LSP lost less weight and had smaller reductions in waist circumference compared with the HSP. Changes in body fat mass, percentage fat mass, fat-free mass and hip circumference did not significantly differ between phenotypes. On test meal days, under HED conditions, the LSP consumed significantly more energy from snacks compared with the HSP. Under LED conditions, EI did not significantly differ between LSP and HSP. Additionally, across conditions, the LSP showed a greater drive for HED foods compared with the HSP who 
showed a preference for LED foods on the LFPQ. The LSP also reported less control over eating and found the weight loss programme more difficult to adhere to compared with the HSP

Lower weight loss in the LSP is consistent with one previous study in men, which reported that the LSP lost less weight over 16 weeks compared with the HSP ${ }^{(15)}$. The differences in weight loss between satiety phenotypes were similar across studies (present study: $-3 \cdot 1 v .-6 \cdot 4 \%$, previous study: -3.3 to $-4 \cdot 3 \% v .-5 \cdot 4$ to $-6.6 \%)$. Thus, the present findings, which extend to women, confirm that the LSP is linked with poorer weight loss outcomes and extend to women. Yet, not all studies reported that the LSP is linked with less weight loss, with one study reporting no effects $^{(22)}$. To explain the mixed findings it has been suggested that the LSP may be particularly influential when participants are following a satiating diet and less influential when the LSP are following an energy-restricted diet $^{(22)}$. The present findings do not add support to this explanation as some participants were following an energy-restricted programme. Therefore, while the present study reported effects in a women-only sample, it remains unclear which aspects of the sample or programme may affect the extent to which the LSP will influence weight loss. Nevertheless, the impact of the LSP on appetite control and weight loss reported here is consistent with previous research highlighting that managing appetite control is one of the main challenges to weight $\operatorname{loss}^{(7)}$. The present findings extend previous research by confirming that there are particular individuals who are least able to detect sensations of fullness and ultimately have greater difficulty losing weight. This finding has important implications for weight management strategies. For example, weight management programmes could screen participants in the early phases of the programme to identify individuals who report a weak ability to detect fullness sensations and offer additional support or dietary strategies that promote satiety (e.g. low energy-density strategies) to optimise weight loss. Future research should assess whether such additional support provided to the LSP can optimise weight loss in this group.

However, it is also important to note that there were no significant changes in body composition between the LSP and HSP. The lack of significant differences in body composition could be due to a low sample size because body composition data could not be collected for a sub-sample of participants. It could also be due to body fat being measured in week 2 and not at the start of the weight loss programme. The weight change (\%) at week 2 was a significant covariate of weight change and changes in fat mass and percentage body fat at the end of the programme. Thus, significant differences between phenotypes for changes in body fat might have been observed if it had been possible to assess body fat at the start of the weight loss programme.

Findings from the test meal days suggest that the lower weight loss in the LSP was due to weaker appetite control. The LSP exhibited a greater drive for HED foods and under HED conditions consumed more snacks (kJ) compared with the HSP. This corroborates previous research which reported that the LSP exhibited a greater drive for high-fat foods and consumed more energy compared with the $\mathrm{HSP}^{(11)}$. Other research has also shown that the LSP shows psychological characteristics linked with overeating such as greater night eating symptoms, external hunger ${ }^{(14)}$ and trait disinhibi$\operatorname{tion}^{(10,11)}$. Moreover, in the present study during the weight loss programme, the LSP reported less control over eating and more difficulty adhering to the programme compared with the HSP. It seems that for the LSP, detecting fullness sensations and controlling EI is more challenging compared with the HSP, and over time this leads to less weight loss. These findings are important because while previous research has shown that the LSP is linked to less weight loss, the present study provides support that the inferior weight loss is due to weaker appetite control in LSP, as indicated by objective and self-report measures. Of note, unlike previous research ${ }^{(10,11)}$ the LSP did not score significantly higher on trait disinhibition compared with the HSP. While there was a trend for the LSP to score higher compared with the HSP, this may not have been significant because trait disinhibition was measured at week 2 of the weight loss programme. Trait disinhibition can decrease during weight loss attempts ${ }^{(32)}$, thus it might be that measuring trait disinhibition at week 2, rather than at the start of the programme, minimised the opportunity to observe significant differences between satiety phenotypes. Additionally, the food diaries did not reveal differences in self-reported intake (possibly due to underreporting and imprecision of self-reported dietary intakes $\left.{ }^{(33,34)}\right)$. But the lack of differences in objectively assessed physical activity and sleep duration adds support that the differences in weight loss between satiety phenotypes were attributable to LSP's weaker appetite control.

For the first time, the present study compared LSP's and HSP's appetite response to meals varying in energy density. Previous research has only examined appetite responses in the satiety phenotypes to one type of meal, where energy density has not been manipulated ${ }^{(11,14)}$. The present findings showed that the LSP only consumed greater EI compared with the HSP when consuming HED foods, not LED foods. Thus, the LSP may be most susceptible to overconsumption when consuming HED foods, while LED foods can prevent excessive EI in LSP. This has important implications for our obesogenic environment where energy-dense foods are readily available ${ }^{(35)}$. Indeed, under LED conditions, the LSP consumed more grams of food compared with the HSP, but evening meal and snack EI did not differ. These findings suggest that LED meals provide an effective strategy for the LSP to eat larger quantities of food without consuming excessive energy.

Interestingly, at the start of the trial the LSP had greater resting systolic and diastolic blood pressure compared with the HSP (albeit, average values were still within clinically normal ranges ${ }^{(36)}$ ), even after controlling for the starting body weight and BMI. As far as we are aware, no other studies have reported differences in blood pressure between the satiety phenotypes. Caution is needed interpreting this difference as blood pressure can vary due to a number of factors beyond satiety phenotypes, but greater blood pressure is consistent with the characteristics of the LSP or low satiating efficiency profiles that previous studies have identified. For instance, stress, intake of high-fat food, overconsumption and shorter sleep durations are factors associated with high blood pressure that previous research has identified in the $\operatorname{LSP}^{(11-14,37)}$. More research is needed to support and explain 
this finding, but it indicates that the LSP may be associated with wider health implications.

There are a number of limitations with the present study which mean the findings should be interpreted with caution. First, due to restrictions in accessing and recruiting volunteers, the study could not obtain baseline appetite measures prior to engagement in the Slimming World, UK or NHS Live Well weight loss programmes. This is especially of concern because participants were recruited from two different weight loss programmes. While, prior percentage weight change during the programme (and programme type) was controlled for in the analyses, it remains possible that the first weeks of the programmes affected appetite responses and the satiety phenotype grouping rather than the grouping being based on underlying appetite traits per se. Therefore, study findings need to be interpreted with caution, and future research should include true baseline appetite measures and recruit from one weight loss programme to confirm the role of satiety phenotypes on weight loss. It is also important to note that tertile splits were conducted on the data, meaning that twenty-seven unclassified participants were not included in the data analyses. Tertile splits were used to be consistent with previous research to allow for cross-study comparisons. However, even though an ANCOVA identified raw SQ scores as a significant covariate on body weight change, it is not clear whether the estimated effect applied to the unclassified group. This is important as the unclassified group also had a BMI classified overweight or obese, and research needs to identify effective strategies for weight management for this group as well as for the LSP. The study design was also limited by the absence of a control group not engaged in weight loss. It would be useful to compare weight changes, food preferences and food intake in response to energy-density manipulations in a group not engaged in weight loss. Also, the ad libitum meals provided access to only LED or HED foods. The LSP might have opted for HED foods if they were available in the LED conditions, especially as the LSP showed a high drive for HED foods across both conditions as measured by the LFPQ. Further research could provide a selection of LED and HED food options at the ad libitum evening meal and assess food choice and intake. Methods to assess weight also varied, with participants being weighed on scales during week 1 and weighed under standardised conditions (fasted) using air plethysmography in weeks 2 and 14. However, all participants underwent these mixed methods of assessment and as such, the resulting variance was unlikely to have differed between the satiety phenotypes. Additionally, appetite control was assessed behaviourally and it would be useful for future research to incorporate biomarkers of appetite control to further characterise the LSP and HSP. Menstrual phase (date of last cycle and average cycle length) was assessed during study screening and of the completed responses; at the start of the weight management programme, there did not appear to be a difference in the number of LSP and HSP in the follicular or luteal phase. However, a number of participants did not provide complete answers or reported either irregular or no menstruation ( $n$ 30), meaning no formal analyses on these data could be reported. Therefore, future studies should collect more information on menstrual phase and control for its possible influence on appetite control on the test meal days and weight change ${ }^{(38,39)}$. Finally, the study was slightly underpowered by two participants and the body composition analyses were conducted on a sub-sample of participants. As such, replication of these study findings in larger samples and different populations, along with systematic reviews and meta-analyses of multiple studies are recommended before informed conclusions can be drawn about the impact of satiety phenotypes on weight loss.

\section{Conclusion}

The ability to resist the drive to eat varies from person to person. This can be measured by the strength of satiety responsiveness. Low-satiety responsiveness is detrimental for weight loss but LED dietary strategies may improve appetite control in the LSP. Further research exploring these satiety behavioural phenotypes is highly warranted.

\section{Acknowledgements}

This trial was registered on ClinicalTrials.gov no. NCT02012426. We thank the women who participated in this trial. We are also grateful to Dr Stephen Whybrow (University of Aberdeen) for analysing the food diaries. The present data sets are available from the corresponding author on reasonable request.

The trial was funded by Slimming World UK. Slimming World UK supported the design of the test meals and recruitment. The funder had no role in the analysis or writing of this article.

N. B., J. B., J. S. and G. F. designed the research; J. L. supported the design of the meals and recruitment; N. B., D. C. and F. C. conducted the trial; A. M. integrated and processed the physical activity data. N. B. performed statistical analyses and wrote the manuscript. All authors read and approved the final manuscript.

J. L. is employed by Slimming World, UK; J. S. consults for Slimming World through the University of Leeds consulting service. All other authors have no conflicts of interest.

\section{Supplementary material}

For supplementary material/s referred to in this article, please visit https://doi.org/10.1017/S000711451900179X

\section{References}

1. Public Health England (2017) Health matters: obesity and the food environment 2017. https://www.gov.uk/government/ publications/health-matters-obesity-and-the-food-environment/ health-matters-obesity-and-the-food-environment-2 (accessed June 2017).

2. Wardle J \& Johnson F (2002) Weight and dieting: examining levels of weight concern in British adults. Int J Obes 26, 1144-1149.

3. Teixeira PJ, Going SB, Sardinha LB, et al. (2005) A review of psychosocial pre-treatment predictors of weight control. Obes $\operatorname{Rev}$ 6, 43-65.

4. Crawford D, Jeffery RW \& French SA (2000) Can anyone successfully control their weight? Findings of a three year community-based study of men and women. Int J Obes $\mathbf{2 4}$, 1107-1110. 
5. Wing RR \& Hill JO (2001) Successful weight loss maintenance. Annu Rev Nutr 21, 323-341.

6. Mann T, Tomiyama AJ, Westling E, et al. (2007) Medicare's search for effective obesity treatments: diets are not the answer. Am Psychol 62, 220-233.

7. Stubbs J, Brogelli D, Pallister C, et al. (2012) Behavioural and motivational factors associated with weight loss and maintenance in a commercial weight management programme. Open Obes J 4, 35-43.

8. Womble LG, Williamson DA, Greenway FL, et al. (2001) Psychological and behavioral predictors of weight loss during drug treatment for obesity. Int J Obes 25, 340-345.

9. Drapeau V, Hetherington M \& Tremblay A (2011) Impact of eating and lifestyle behaviors on body weight: beyond energy value. In Handbook of Behavior, Food and Nutrition, pp. 693-706 [VR Preedy, RR Watson and CR Martin, editors]. New York: Springer.

10. Barkeling B, King NA, Naslund E, et al. (2007) Characterization of obese individuals who claim to detect no relationship between their eating pattern and sensations of hunger or fullness. Int J Obes 31, 435-439.

11. Dalton M, Hollingworth S, Blundell J, et al. (2015) Weak satiety responsiveness is a reliable trait associated with hedonic risk factors for overeating among women. Nutrients 7, 7421-7436.

12. Drapeau V, Blundell J, Therrien F, et al. (2005) Appetite sensations as a marker of overall intake. BrJ Nutr 93, 273-280.

13. Drapeau V, King N, Hetherington M, et al. (2007) Appetite sensations and satiety quotient: predictors of energy intake and weight loss. Appetite 48, 159-166.

14. Drapeau V, Blundell J, Gallant AR, et al. (2013) Behavioural and metabolic characterisation of the low satiety phenotype. Appetite 70, 67-72.

15. Arguin H, Tremblay A, Blundell JE, et al. (2017) Impact of a non-restrictive satiating diet on anthropometrics, satiety responsiveness and eating behaviour traits in obese men displaying a high or a low satiety phenotype. BrJ Nutr 118, $750-760$

16. Finlayson G, King N \& Blundell J (2008) The role of implicit wanting in relation to explicit liking and wanting for food: implications for appetite control. Appetite 50, 120-127.

17. Stubbs J, Ferres S \& Horgan G (2000) Energy density of foods: effects on energy intake. Crit Rev Food Sci Nutr 40, 481-515.

18. Bell EA \& Rolls BJ (2001) Energy density of foods affects energy intake across multiple levels of fat content in lean and obese women. Am J Clin Nutr 73, 1010-1018.

19. Buckland NJ, Camidge D, Croden F, et al. (2018) A low energydense diet in the context of a weight-management program affects appetite control in overweight and obese women. J Nutr 148, 798-806.

20. Epstein LH, Truesdale R, Wojcik A, et al. (2003) Effects of deprivation on hedonics and reinforcing value of food. Physiol Behav 78, 221-227.

21. Goldstone AP, de Hernandez CGP, Beaver JD, et al. (2009) Fasting biases brain reward systems towards high-calorie foods. Eur J Neurosci 30, 1625-1635.

22. Drapeau V, Jacob R, Panahi S, et al. (2019) Effect of energy restriction on eating behavior traits and psychobehavioral factors in the low satiety phenotype. Nutrients 11, 245.
23. Faul F, Erdfelder E, Lang A-G, et al. (2007) G*Power 3: a flexible statistical power analysis program for the social, behavioral, and biomedical sciences. Behav Res Methods 39, 175-191.

24. Stubbs RJ, Morris L, Pallister C, et al. (2015) Weight outcomes audit in 1.3 million adults during their first 3 months' attendance in a commercial weight management programme. BMC Public Health 15, 882.

25. NHS-Choices (2015) Start the NHS weight loss plan. http:// www.nhs.uk/Livewell/weight-loss-guide/Pages/losing-weightgetting-started.aspx (accessed January 2014).

26. Stunkard AJ \& Messick S (1985) The three-factor eating questionnaire to measure dietary restraint, disinhibition and hunger. J Psychosom Res 29, 71-83.

27. Blundell J, de Graaf C, Hulshof T, et al. (2010) Appetite control: methodological aspects of the evaluation of foods. Obes Rev $\mathbf{1 1}$, 251-270.

28. Myers A, Gibbons C, Finlayson G, et al. (2017) Associations among sedentary and active behaviours, body fat and appetite dysregulation: investigating the myth of physical inactivity and obesity. BrJ Sports Med 51, 1540-1544.

29. Finglas PM, Roe MA, Pinchen HM, et al. (2015) McCance and Widdowson's The Composition of Foods, 7th summary ed. Cambridge: Royal Society of Chemistry.

30. Elobeid MA, Padilla MA, McVie T, et al. (2009) Missing data in randomized clinical trials for weight loss: scope of the problem, state of the field, and performance of statistical methods. PLOS ONE 4, e6624.

31. Field A (2005) Discovering Statistics using SPSS (Introducing Statistical Methods series), 2nd ed. London: Sage Publications Ltd.

32. Butryn ML, Thomas JG \& Lowe MR (2009) Reductions in internal disinhibition during weight loss predict better weight loss maintenance. Obesity 17, 1101-1103.

33. Poppitt SD, Swann D, Black AE, et al. (1998) Assessment of selective under-reporting of food intake by both obese and non-obese women in a metabolic facility. Int $J$ Obes $\mathbf{2 2}$, 303-311.

34. Heitmann BL \& Lissner L (1995) Dietary underreporting by obese individuals-is it specific or non-specific? Br Med J 311, 986-989.

35. Wadden TA, Brownell KD \& Foster GD (2002) Obesity: responding to the global epidemic. J Consult Clin Psychol 70, 510-525.

36. National Health Service (2016) High blood pressure (hypertension). https://www.nhs.uk/conditions/high-blood-pressurehypertension/ (accessed June 2017).

37. McNeil J, Drapeau V, Gallant AR, et al. (2013) Short sleep duration is associated with a lower mean satiety quotient in overweight and obese men. Eur J Clin Nutr 67, 1328-1330.

38. Geiker NRW, Ritz C, Pedersen SD, et al. (2016) A weight-loss program adapted to the menstrual cycle increases weight loss in healthy, overweight, premenopausal women: a 6-mo randomized controlled trial. Am J Clin Nutr 104, 15-20.

39. Brennan IM, Feltrin KL, Nair NS, et al. (2009) Effects of the phases of the menstrual cycle on gastric emptying, glycemia, plasma GLP-1 and insulin, and energy intake in healthy lean women. Am J Physiol Gastrointest Liver Physiol 297, G602-G610. 